PDES, SUBMANIFOLDS AND

AFFINE DIFFERENTIAL GEOMETRY

BANACH CENTER PUBLICATIONS, VOLUME 57

INSTITUTE OF MATHEMATICS

POLISH ACADEMY OF SCIENCES

WARSZAWA 2002

\title{
DARBOUX TRANSFORMS OF DUPIN SURFACES
}

\author{
EMILIO MUSSO \\ Dipartimento di Matematica Pura ed Applicata, Università di L'Aquila \\ Via Vetoio, I-67100 L'Aquila, Italy \\ E-mail: musso@univaq.it \\ LORENZO NICOLODI \\ Dipartimento di Matematica, Università di Parma \\ Via D'Azeglio 85/A, I-43100 Parma, Italy \\ E-mail: nicolodi@mat.uniroma1.it
}

\begin{abstract}
We present a Möbius invariant construction of the Darboux transformation for isothermic surfaces by the method of moving frames and use it to give a complete classification of the Darboux transforms of Dupin surfaces.
\end{abstract}

1. Introduction. The transformation theory of isothermic surfaces was intensively studied at the turn of the 20th century and in recent years has received a renewed attention because of its connection with the theory of integrable systems. The methods of integrable systems theory made their first appearance in the study of isothermic surfaces with Cieśliński-Goldstein-Sym's zero-curvature formulation of the Gauss-Codazzi equations of an isothermic surface [13]. This work was taken up by Burstall-Hertrich-JerominPedit-Pinkall [8], who described the integrable system of isothermic surfaces in the context of Möbius geometry as an example of the curved flat system of Ferus-Pedit [16]; an equivalent description was given in [6]. This approach provided a coherent framework for discussing the classical transformations of isothermic surfaces. For recent accounts on the transformation theory of isothermic surfaces, we refer the reader to [18, 19, 20], and [7], where the Darboux transformation is described using the loop group formulation according to the general theory of Bäcklund transformations due to Terng-Uhlenbeck [26].

2000 Mathematics Subject Classification: Primary 53A30, 53A05; Secondary 35Q51.

Key words and phrases: isothermic surfaces, Darboux transformation, Dupin surfaces, Ttransformation, Bäcklund transformation.

This research was partially supported by the MURST project Proprietà Geometriche delle Varietà Reali e Complesse, by the GNSAGA of the CNR, and by the European Contract Human Potential Programme, Research Training Network HPRN-CT-2000-00101 (EDGE).

The paper is in final form and no version of it will be published elsewhere. 
The Darboux transformation of isothermic surfaces is defined by realizing them as the focal surfaces of a 2-sphere congruence such that the correspondence induced between the two envelopes preserves the curvature lines and is conformal. The integrability theorem says that for a given isothermic surface there exist infinitely many Darboux transforms [15]. Bianchi [3] proved a permutability theorem for this transformation and introduced a related spectral transformation, the T-transformation [4], which was independently discovered also by Calapso [10].

This paper presents a Möbius invariant construction of the Darboux transformation by the method of moving frames. In this approach the T-transform plays a fundamental role in the sense that the Darboux transform is computed algebraically, without quadratures, starting from the T-transform. The latter is computed by solving the Maurer-Cartan equations for the associated spectral frame. The general construction is applied to discuss the classical formulae for the Darboux transformation in the context of Möbius geometry and, as a main result, to determine explicit formulae for the Darboux transforms of Dupin cyclides. These together with the isothermic surfaces of spherical type, studied by the authors and U. Hertrich-Jeromin in [20], will provide a complete classification of Darboux transforms of Dupin surfaces (see Example 3.2). In our discussion, Dupin cyclides arise as T-transforms of a circular cylinder (a constant mean curvature cylinder). Finally, as another application of the construction we establish a superposition formula for iterated Darboux transforms and prove Bianchi's permutability theorem. This will be useful for the explicit calculation of multisoliton surfaces (see [22]).

H. Bernstein [2], by carrying out a detailed analysis of the Darboux transforms of the standard torus of revolution, has been able to construct new explicit examples of isothermic tori which are not conformally equivalent to the known examples, such as tori of revolution and constant mean curvature tori in space forms [23]. These tori have spherical curvature lines and can develop umbilics, either isolated umbilics or curves of umbilics. Much of the background material used by Bernstein in her work is presented in this paper. In [22], by the same methods, the authors study the Darboux transforms of constant mean curvature surfaces in space forms and their relations with the special isothermic surfaces of Darboux [3]. For the Euclidean and hyperbolic cases see also [19, 20]. That there exist constant mean curvature Darboux transforms of a constant mean curvature surface is a classical result of Bianchi [3], [19]. The particular case of Darboux transforms of the cylinder which have constant mean curvature in Euclidean space has been considered in [19]. These Darboux transforms are related to Sterling-Wente's constant mean curvature multibubbletons [25] (see also Section 4).

Section 2 deals with some basic material. Section 3 presents the construction of the Darboux transformation by the method of moving frames, and Section 4 computes the Darboux transforms of Dupin cyclides. Finally, Section 5 proves a superposition formula and the permutability theorem for iterated Darboux transforms.

Acknowledgments. Part of the material presented here has been circulating for some time as informal notes on conformal geometry and isothermic surfaces written by the authors. We thank Holly Bernstein for reading the original notes and making a number of valuable remarks. We also like to thank Fran Burstall and Udo Hertrich-Jeromin for 
several interesting and pleasant conversations on the subject over the past years. Finally, we take this opportunity to thank the referee for his useful comments and suggestions.

2. Isothermic immersions. Consider Minkowski 5-space $\mathbf{R}_{1}^{5}$ with linear coordinates $x^{0}, \ldots, x^{4}$ and Lorentz scalar product given by the quadratic form

$$
\langle x, x\rangle=-x^{0} x^{4}+\left(x^{1}\right)^{2}+\left(x^{2}\right)^{2}+\left(x^{3}\right)^{2}=\eta_{i j} x^{i} x^{j}
$$

an orientation for which $d x^{0} \wedge \cdots \wedge d x^{4}>0$; and a time-orientation defined by the positive light-cone $\mathcal{L}^{+}=\left\{x \in \mathbf{R}_{1}^{5}:\langle x, x\rangle=0, x^{0}+x^{4}>0\right\}$. Classically, the Möbius space $\mathbf{S}^{3}$ (conformal 3-sphere) is realized as the projective quadric $\left\{[x] \in \mathbf{R P}^{4}:\langle x, x\rangle=0\right\}$. Accordingly, $\mathbf{S}^{3}$ inherits a natural conformal structure and the identity component $G$ of the pseudo-orthogonal group of (1) acts transitively on $\mathbf{S}^{3}$ as group of orientationpreserving, conformal transformations (see $[12,9]$ ). In this model for Möbius geometry, the de Sitter space $\mathbf{S}_{1}^{4}=\left\{y \in \mathbf{R}_{1}^{5}:\langle y, y\rangle=1\right\}$ parametrizes oriented 2-spheres in $\mathbf{S}^{3}$ by $y \mapsto y^{\perp} \cap \mathbf{S}^{3}$, and is acted on by $G$. A Möbius frame is a basis $\left(g_{0}, \ldots, g_{4}\right)$ of $\mathbf{R}_{1}^{5}$ such that $g_{i}=g \epsilon_{i}$, for $g \in G, \epsilon_{0}, \ldots, \epsilon_{4}$ standard basis of $\mathbf{R}_{1}^{5}$. Geometrically, the unit space-like vectors $g_{1}, g_{2}, g_{3}$ represent 2 -spheres which intersect orthogonally, and $\left[g_{0}\right],\left[g_{4}\right] \in \mathbf{S}^{3}$ their intersection points. Regarding $g_{0}, \ldots, g_{4}$ as $\mathbf{R}^{5}$-valued functions defined on $G$ there exist unique 1-forms $\left\{\omega_{j}^{i}\right\}_{0 \leq i, j \leq 4}$ such that

$$
d g_{i}=\omega_{i}^{j} g_{j}, \quad \omega_{i}^{k} \eta_{k j}+\omega_{j}^{k} \eta_{k i}=0
$$

and satisfying the structure equations

$$
d \omega_{j}^{i}=-\omega_{k}^{i} \wedge \omega_{j}^{k}
$$

$\omega=\left(\omega_{j}^{i}\right)=g^{-1} d g$ is the Maurer-Cartan form of $G$.

Definition 2.1. Consider $\mathbf{S}^{3}$ endowed with the standard round metric $g_{0}$, and let $\mathcal{U} \subset \mathbf{R}^{2}$ a simply connected domain with preferred coordinates $(x, y)$. A smooth immersion $f: \mathcal{U} \rightarrow \mathbf{S}^{3}$ with no umbilic points is called isothermic if $(x, y)$ are conformal curvature line coordinates for the induced Riemannian metric $d s_{f}^{2}$.

The notion of isothermic immersion is conformally invariant, that is, it only depends on the conformal class $\left[g_{0}\right]$ of the metric on $\mathbf{S}^{3}$. If $a$ and $c$ denote the principal curvatures along the $x$ and $y$-directions, respectively, then there exists a function $\Phi$ such that

$$
\Phi^{2}\left(d x^{2}+d y^{2}\right)=\frac{1}{4}(a-c)^{2} d s_{f}^{2} .
$$

$\Phi$ is called the Calapso potential of the isothermic immersion and is a conformal invariant of the immersion. It is uniquely defined up to the choice of conformal curvature line coordinates. Geometrically, $\Phi^{2}$ is the conformal factor relating the metric induced by the central sphere congruence (Gauss conformal map) of $f$ to the metric $d x^{2}+d y^{2}$ (see for example [8]).

2.1. Principal frames. A Möbius frame field along a conformal immersion $f: \mathcal{U} \rightarrow \mathbf{S}^{3}$ is a smooth map $\mathbf{a}=\left(a_{0}, \ldots, a_{4}\right): \mathcal{U} \rightarrow G$ such that $f(p)=\left[a_{0}\right](p)$, for all $p \in \mathcal{U}$. For any such a field, let $\alpha=\left(\alpha_{j}^{i}\right)=\mathbf{a}^{*} \omega$. We say that $\mathbf{a}$ is a principal frame field along $f$ if

$$
\alpha_{0}^{3}=0
$$




$$
\begin{aligned}
& \alpha_{1}^{3} \wedge \alpha_{0}^{1}=\alpha_{2}^{3} \wedge \alpha_{0}^{2}=0 \\
& \alpha_{0}^{1} \wedge \alpha_{0}^{2} \neq 0
\end{aligned}
$$

If $f$ is isothermic, then

$$
\alpha_{0}^{1} \wedge d x=\alpha_{0}^{2} \wedge d y=0
$$

and there exist smooth functions $l_{1}, l_{2}$ such that $\alpha_{1}^{2}=l_{2} \alpha_{0}^{1}-l_{1} \alpha_{0}^{2}$. It is not difficult to see that

$$
d\left(\alpha_{0}^{0}-l_{1} \alpha_{0}^{1}-l_{2} \alpha_{0}^{2}\right)=0 .
$$

REMARK 2.2. Principal frames may be defined along any smooth immersion $f: \mathcal{U} \rightarrow$ $\mathbf{S}^{3}$ with no umbilic points. (3) is a necessary and sufficient condition for the existence of a reparametrization $h: \mathcal{U} \rightarrow \mathcal{U}$ of $\mathcal{U}$ such that $f \circ h$ is an isothermic immersion.

The totality of principal frames along an isothermic immersion $f$ is a smooth bundle $\pi_{f}: \mathcal{P}(f) \rightarrow \mathcal{U}$ whose structure group is diffeomorphic to the group of all elements

$$
g^{+}(V)=\left(\begin{array}{ccc}
\left(v^{4}\right)^{-1} & \left(v^{4}\right)^{-1} t v & v^{0} \\
0 & I_{3} & v \\
0 & 0 & v^{4}
\end{array}\right), \quad v={ }^{t}\left(v^{1}, v^{2}, v^{3}\right)
$$

$V \in \mathcal{L}^{+}$. If we think of $\mathcal{P}(f)$ as a submanifold of $G$, then the Maurer-Cartan form $\omega$ of $G$ restricted to $\mathcal{P}(f)$ satisfies

$$
\begin{aligned}
& \omega_{0}^{3}=0, \quad \omega_{0}^{1}=e^{u} d x, \quad \omega_{0}^{2}=e^{u} d y, \\
& \omega_{1}^{3}=H_{1} e^{u} d x, \quad \omega_{2}^{3}=H_{2} e^{u} d y,
\end{aligned}
$$

where $u, H_{1}, H_{2}$ are smooth real-valued functions defined on the total space $\mathcal{P}(f)$. A straightforward calculation yields

$$
e^{u\left(\mathbf{b} \cdot g^{+}(V)\right)}=\frac{1}{v^{4}} e^{u(\mathbf{b})}, \quad H_{j}\left(\mathbf{b} \cdot g^{+}(V)\right)=v^{4} H_{j}(\mathbf{b})-v^{3}, \quad j=1,2,
$$

for each $\mathbf{b} \in \mathcal{P}(f)$, and for each $g^{+}(V)$. This tells us that, wherever $H_{1}(\mathbf{b})-H_{2}(\mathbf{b}) \neq 0$ (that is away from umbilic points), the real-valued function $\tilde{\Phi}=\frac{1}{2}\left(H_{1}-H_{2}\right) e^{u}: \mathcal{P}(f) \rightarrow$ $\mathbf{R}$ is constant along the fibers of $\pi_{f}$. The function $\Phi: \mathcal{U} \rightarrow \mathbf{R}$ defined by $\tilde{\Phi}=\Phi \circ \pi_{f}$ coincides with the Calapso potential of the isothermic immersion $f$.

EXAMPLE 2.3 (Euclidean frame). The Euclidean group of rigid motions embeds canonically in the Möbius group $G$ and $\mathbf{E}^{3}$ is viewed as $\mathbf{S}^{3}$ minus the point at infinity $\left[\epsilon_{4}\right]=\left[{ }^{t}(0,0,0,0,1)\right]$. Let $f: \mathcal{U} \rightarrow \mathbf{E}^{3}$ be an isothermic immersion and consider the principal Euclidean framing $\mathbf{e}: \mathcal{U} \rightarrow \mathbf{E}(3)$ along $f$ given by

$$
\mathbf{e}=\left(f ; \frac{f_{x}}{\left\|f_{x}\right\|}, \frac{f_{y}}{\left\|f_{y}\right\|}, \frac{f_{x} \times f_{y}}{\left\|f_{x} \times f_{y}\right\|}\right) .
$$

Set $\left\|f_{x}\right\|=\left\|f_{y}\right\|=e^{\varphi}$ and let $a$ and $c$ be the principal curvature of $f$ with respect to e. Considering e as a map into $\mathbf{E}(3) \subset G$, its Maurer-Cartan form $\eta=\left(\eta_{j}^{i}\right)$ becomes 


$$
\eta=\left(\begin{array}{ccccc}
0 & 0 & 0 & 0 & 0 \\
e^{\varphi} d x & 0 & \varphi_{y} d x-\varphi_{x} d y & -a e^{\varphi} d x & 0 \\
e^{\varphi} d y & -\varphi_{y} d x+\varphi_{x} d y & 0 & -c e^{\varphi} d y & 0 \\
0 & a e^{\varphi} d x & c e^{\varphi} d y & 0 & 0 \\
0 & e^{\varphi} d x & e^{\varphi} d y & 0 & 0
\end{array}\right)
$$

In terms of the Euclidean data, the Calapso potential is given by $\Phi=\frac{1}{2}(a-c) e^{\varphi}$.

EXAmple 2.4 (Central frame). Let $f: \mathcal{U} \subset \mathbf{R}^{2} \rightarrow \mathbf{S}^{3}$ be an immersion with no umbilic points. Then there exists a frame $\mathbf{b}: \mathcal{U} \rightarrow G$ along $f$ (cf. [9]), the central frame, with connection form $\beta$ given by

$$
\left(\begin{array}{ccccc}
-2 q_{2} \beta_{0}^{1}+2 q_{1} \beta_{0}^{2} & p_{1} \beta_{0}^{1}+p_{2} \beta_{0}^{2} & -p_{2} \beta_{0}^{1}+p_{3} \beta_{0}^{2} & 0 & 0 \\
\beta_{0}^{1} & 0 & -q_{1} \beta_{0}^{1}-q_{2} \beta^{2} 0 & -\beta_{0}^{1} & p_{1} \beta_{0}^{1}+p_{2} \beta_{0}^{2} \\
\beta_{0}^{2} & q_{1} \beta_{0}^{1}+q_{2} \beta_{0}^{2} & 0 & \beta_{0}^{2} & -p_{2} \beta_{0}^{1}+p_{3} \beta_{0}^{2} \\
0 & \beta_{0}^{1} & -\beta_{0}^{2} & 0 & 0 \\
0 & \beta^{1} 0 & \beta_{0}^{2} & 0 & 2 q_{2} \beta_{0}^{1}-2 q_{1} \beta_{0}^{2}
\end{array}\right),
$$

such that $\beta_{0}^{1} \wedge d x=\beta_{0}^{2} \wedge d y=0, \beta_{0}^{1} \wedge \beta_{0}^{2} \neq 0$. The smooth functions $p_{1}, p_{2}, p_{3}, q_{1}, q_{2}$ form a complete system of conformal invariants for $f$ and satisfy the following structure equations:

$$
\begin{aligned}
& d \beta_{0}^{1}=-q_{1} \beta_{0}^{1} \wedge \beta_{0}^{2}, d \beta_{0}^{2}=-q_{2} \beta_{0}^{1} \wedge \beta_{0}^{2}, \\
& d q_{1} \wedge \beta_{0}^{1}+d q_{2} \wedge \beta_{0}^{2}=\left(1+p_{1}+p_{3}+q_{1}^{2}+q_{2}^{2}\right) \beta_{0}^{1} \wedge \beta_{0}^{2}, \\
& d q_{2} \wedge \beta_{0}^{1}-d q_{1} \wedge \beta_{0}^{2}=-p_{2} \beta_{0}^{1} \wedge \beta_{0}^{2}, \\
& d p_{1} \wedge \beta_{0}^{1}+d p_{2} \wedge \beta_{0}^{2}=\left(4 q_{2} p_{2}+q_{1}\left(3 p_{1}+p_{3}\right)\right) \beta_{0}^{1} \wedge \beta_{0}^{2}, \\
& d p_{2} \wedge \beta_{0}^{1}-d p_{3} \wedge \beta_{0}^{2}=\left(4 q_{1} p_{2}-q_{2}\left(p_{1}+3 p_{3}\right)\right) \beta_{0}^{1} \wedge \beta_{0}^{2} .
\end{aligned}
$$

If $\mathbf{b}=\left(b_{i}\right)_{i=0}^{4}$ is a central frame, any other central frame is given by $\left(b_{0},-b_{1},-b_{2}, b_{3}, b_{4}\right)$. The mapping $b_{3}: \mathcal{U} \rightarrow \mathbf{S}_{1}^{4}$ coincides with the central sphere congruence (Gauss conformal map) of $f$. Its second envelope $\left[b_{4}\right]: \mathcal{U} \rightarrow \mathbf{S}^{3}$ is called the conformal transform of $f$.

Let $\mathbf{e}=\left(e_{0}, \ldots, e_{4}\right): \mathcal{U} \rightarrow \mathbf{E}(3) \subset G$ be a Euclidean principal framing along $f$ and denote by $\eta=\left(\eta_{j}^{i}\right)$ its Maurer-Cartan form. Setting $H=\frac{1}{2}(a+c), R=\frac{1}{2}(a-c)$, and $d H=R\left(u \eta_{0}^{1}+v \eta_{0}^{2}\right)$, we may adapt $\mathbf{e}$ to a central framing $\mathbf{b}=\left(b_{0}, \ldots, b_{4}\right)$, where

$$
\begin{aligned}
& b_{0}=R e_{0}, \quad b_{1}=e_{1}-u e_{0}, \\
& b_{2}=e_{2}+v e_{0}, \quad b_{3}=e_{3}+H e_{0}, \\
& b_{4}=\frac{1}{R}\left\{\frac{1}{2}\left(H^{2}+u^{2}+v^{2}\right) e_{0}-u e_{1}+v e_{2}+H e_{3}+e_{4}\right\} .
\end{aligned}
$$

A direct calculation yields

$$
\begin{gathered}
\beta_{0}^{1}=R \eta_{0}^{1}, \quad \beta_{0}^{2}=R \eta_{0}^{2}, \quad \beta_{0}^{3}=0, \quad \beta_{1}^{3}=\beta_{0}^{1}, \quad \beta_{2}^{3}=-\beta_{0}^{2}, \quad \beta_{3}^{0}=0 \\
\beta_{0}^{0}=\frac{1}{R}\left[a_{1} \beta_{0}^{1}-c_{2} \beta_{0}^{2}\right], \quad \beta_{1}^{2}=-\frac{1}{2 R}\left[c_{2} \beta_{0}^{1}+a_{1} \beta_{0}^{2}\right], \\
\beta_{1}^{0}=\frac{1}{R}\left\{\left[\frac{H}{R}\left(\frac{H}{2}-a\right)-u_{1}-\frac{v a_{2}}{2 R}-\frac{u^{2}-v^{2}}{2 R}\right] \beta_{0}^{1}+\left[\frac{u v}{R}-u_{2}-\frac{v c_{1}}{2 R}\right] \beta_{0}^{2}\right\}, \\
\beta_{2}^{0}=\frac{1}{R}\left\{\left[\frac{u v}{R}+v_{1}-\frac{u a_{2}}{2 R}\right] \beta_{0}^{1}+\left[\frac{H}{R}\left(\frac{H}{2}-c\right)+v_{2}-\frac{u c_{1}}{2 R}+\frac{u^{2}-v^{2}}{2 R}\right] \beta_{0}^{2}\right\},
\end{gathered}
$$


where for any function $g, g_{1}$ and $g_{2}$ denote the directional derivatives defined by $d g=$ $g_{1} \beta_{0}^{1}+g_{2} \beta_{0}^{2}$. This allows us to express the set of invariant functions $p_{1}, p_{2}, p_{3}, q_{1}, q_{2}$ in Euclidean terms.

If the immersion $f$ is isothermic, then $p_{2}$ vanishes, and conversely [21]. In this case, there exists a unique central frame such that

$$
\beta_{0}^{1}=\Phi d x, \quad \beta_{0}^{2}=\Phi d y
$$

and $\Phi=: e^{\psi}$ is the Calapso potential of $f$.

2.2. The Calapso equation and the T-transformation. Let $f: \mathcal{U} \subset \mathbf{R}^{2} \rightarrow \mathbf{S}^{3}$ be an isothermic immersion and let us retain the notations of the preceding example. From (5) it follows that $q_{1}=e^{-\psi} \psi_{y}$ and $q_{2}=-e^{-\psi} \psi_{x}$ and (4) becomes

$$
\beta=\left(\begin{array}{ccccc}
2 d \psi & p_{1} e^{\psi} d x & p_{3} e^{\psi} d y & 0 & 0 \\
e^{\psi} d x & 0 & -\psi_{y} d x+\psi_{x} d y & -e^{\psi} d x & p_{1} e^{\psi} d x \\
e^{\psi} d y & \psi_{y} d x-\psi_{x} d y & 0 & e^{\psi} d y & p_{3} e^{\psi} d y \\
0 & e^{\psi} d x & -e^{\psi} d y & 0 & 0 \\
0 & e^{\psi} d x & e^{\psi} d y & 0 & -2 d \psi
\end{array}\right)
$$

Moreover, from equation (6), we get

$$
p_{1}+p_{3}=-1-e^{-2 \psi} \Delta \psi
$$

Substituting into equations (8) and (9) yields

$$
\begin{aligned}
d\left(e^{2 \psi}\left(p_{1}-p_{3}\right)\right)= & -e^{2 \psi}\left\{\left(e^{-2 \psi} \Delta \psi\right)_{x}+4 \psi_{x}\left(1+e^{-2 \psi} \Delta \psi\right)\right\} d x \\
& +e^{2 \psi}\left\{\left(e^{-2 \psi} \Delta \psi\right)_{y}-4 \psi_{y}\left(1+e^{-2 \psi} \Delta \psi\right)\right\} d y
\end{aligned}
$$

The integrability condition of this equation is precisely the Calapso equation ${ }^{1}$

$$
\Delta\left(e^{-\psi}\left(e^{\psi}\right)_{x y}\right)+2\left(e^{2 \psi}\right)_{x y}=0 .
$$

Conversely, if $\Phi=e^{\psi}$ is a solution of (16), the right-hand-side of equation (15) is a closed 1-form, say $\gamma_{\Phi}$. Thus $\gamma_{\Phi}=d H$ for some function $H$ determined up to an additive constant. Define $e^{2 \psi}\left(p_{1}-p_{3}\right)=H+m, m$ any real constant, and set (cf. 14)

$$
\tilde{p}_{1}=p_{1}+m e^{-2 \psi}, \quad \tilde{p}_{3}=p_{3}-m e^{-2 \psi} .
$$

Accordingly, consider the $\mathcal{G}$-valued 1 -forms $\beta(m)$ defined by

$$
\left(\begin{array}{ccccc}
2 d \psi & \left(p_{1} e^{\psi}+m e^{-\psi}\right) d x & \left(p_{3} e^{\psi}-m e^{-\psi}\right) d y & 0 & 0 \\
e^{\psi} d x & 0 & -\psi_{y} d x+\psi_{x} d y & -e^{\psi} d x & \left(p_{1} e^{\psi}+m e^{-\psi}\right) d x \\
e^{\psi} d y & \psi_{y} d x-\psi_{x} d y & 0 & e^{\psi} d y & \left(p_{3} e^{\psi}-m e^{-\psi}\right) d y \\
0 & e^{\psi} d x & -e^{\psi} d y & 0 & 0 \\
0 & e^{\psi} d x & e^{\psi} d y & 0 & -2 d \psi
\end{array}\right) .
$$

The forms $\beta(m)$ satisfies the Maurer-Cartan integrability condition

$$
d \beta(m)=-\beta(m) \wedge \beta(m),
$$

\footnotetext{
${ }^{1}$ The deduction of Calapso's equation follows an argument similar to that in [8]; there a slightly different Möbius invariant frame is considered. The equation named after Calapso was obtained independently by Rothe [24] and Calapso [10] as defining equation for isothermic surfaces.
} 
and then integrates to a map $\mathbf{b}(m): \mathcal{U} \rightarrow G, \mathbf{b}(m)^{-1} d \mathbf{b}(m)=\beta(m)$, which is the central frame of the isothermic immersion $f_{m}=\left[b(m)_{0}\right]$. This 1-parameter family of isothermic immersions amounts to the second order Möbius deformations ${ }^{2}$ of $f[17,12,21]$. So, a solution to Calapso's equation introduces an auxiliary parameter which describes the conformal deformation through isothermic surfaces. All deformations have the same Calapso potential $\Phi$.

Definition 2.5 (T-transformation). Two isothermic immersions $f, \hat{f}: \mathcal{U} \rightarrow \mathbf{S}^{3}$ are said to be T-transforms (spectral deformations) of each other if they have the same Calapso potential. The T-transformation for isothermic surfaces was originally introduced by Calapso [10] and Bianchi [4].

REMARK 2.6. The spectral family $f_{m}$ constructed above describes all T-transforms of $f=f_{0}$. In fact, any umbilic free T-transform of $f$ is Möbius equivalent to $f_{m}$ for some $m \in \mathbf{R}([21,11])$.

Example 2.7 (Dupin surfaces). The Calapso potential of an isothermic immersion $f$ is constant if and only if the invariant functions $q_{1}$ and $q_{2}$ vanish. The vanishing of $q_{1}$ and $q_{2}$ is a necessary and sufficient condition for $f$ being a cyclide of Dupin (cf. Section 4). We recall that an immersion in $\mathbf{S}^{3}$ is a Dupin surface if each principal curvature is constant along any line of curvature tangent to its principal direction. It is known that the only Dupin surfaces in $\mathbf{R}^{3}$ are spheres, planes and the cyclides of Dupin. Examples of Dupin cyclides include tori of revolution, circular cylinders and cones. It turns out that all Dupin cyclides are conformally equivalent to either one of these examples [5, 27]. Section 4 will describe Dupin cyclides as T-transforms of a circular cylinder.

EXAMPLE 2.8 (Willmore isothermic surfaces). In terms of the conformal invariants, Willmore isothermic surfaces are characterized by the equations ${ }^{3}$ (cf. $[9,21]$ )

$$
p_{1}=p_{3}, \quad p_{2}=0 \text {. }
$$

By (8) and (9),

and then

$$
\frac{d p_{1}}{p_{1}}=4\left(q_{2} \beta_{0}^{1}-q_{1} \beta_{0}^{2}\right)=-2 \beta_{0}^{0}
$$

$$
p_{1}=\tilde{c} e^{-4 \psi},
$$

for a constant $\tilde{c}$. But, by (14),

$$
p_{1}=-\frac{1}{2}\left(e^{-2 \psi} \Delta \psi+1\right) .
$$

Then,

$$
\Delta \psi=c e^{-2 \psi}-e^{2 \psi}
$$

\footnotetext{
${ }^{2}$ Two immersions $f, \tilde{f}: \mathcal{U} \rightarrow \mathbf{S}^{3}$ are (second order) G-deformations of each other if there is a smooth map $\mathbf{b}: \mathcal{U} \rightarrow G$ with the property that $\mathbf{b}(p) \cdot \tilde{f}$ and $f$ agree up to order two at $p$, for each $p \in \mathcal{U} . f$ is said deformable if it admits a non-trivial deformation, i.e., a deformation $\tilde{f}$ that is not $G$-equivalent to $f$. The non-trivial deformations of $f$ arise in a 1-parameter family.

${ }^{3}$ The equation $p_{1}=p_{3}$ is equivalent to the Euler-Lagrange equation $\Delta H+2 H\left(H^{2}-K\right)=0$ for the Willmore functional. In fact, using the expressions for $p_{1}$ and $p_{3}$ given by (12) and (13), one computes $p_{3}-p_{1}=\frac{1}{R^{3}}\left(\Delta H+2 H\left(H^{2}-K\right)\right)$.
} 
$c$ a constant. It is easily verified that any solution of (18) satisfies the Calapso equation. Therefore, (18) defines Willmore isothermic surfaces. According as $c$ is positive, negative or zero, the equation (18) accounts, up to conformal equivalence, for the minimal surfaces in 3-dimensional space forms $\mathbf{H}^{3}, \mathbf{S}^{3}, \mathbf{R}^{3}$, respectively [5]. In [20, 11], it has been proved that the T-transforms of minimal immersions in 3-dimensional space forms are locally conformally equivalent to constant mean curvature surfaces in space forms and that all constant mean curvature surfaces in space forms arise in this way.

\section{The Darboux transformation by moving frames}

Definition 3.1 (Darboux transformation). A Darboux isothermic frame or a curved flat frame $e^{4}$ is a principal frame $\mathbf{a}: \mathcal{U} \rightarrow G$ along an isothermic immersion $f: \mathcal{U} \rightarrow \mathbf{S}^{3}$ such that

$$
\begin{aligned}
\left(-\alpha_{1}^{0}+i \alpha_{2}^{0}\right) \wedge\left(\alpha_{0}^{1}+i \alpha_{0}^{2}\right) & =0 \\
\alpha_{3}^{0} & =0 \\
\alpha_{1}^{0} \wedge \alpha_{2}^{0} & \neq 0 .
\end{aligned}
$$

If $\mathbf{a}$ is a curved flat frame, then $\left(a_{4},-a_{1}, a_{2}, a_{3}, a_{0}\right)$ defines a principal frame along $\hat{f}:=$ $\left[a_{4}\right]$ and $(x, y)$ are conformal curvature line coordinates for $\hat{f}$. Thus $\hat{f}$ is another isothermic immersion. Moreover, the mapping $\sigma:=a_{3}: \mathcal{U} \rightarrow \mathbf{S}_{1}^{4}$ defines a sphere congruence whose envelopes are $f$ and $\hat{f}$. The correspondence induced by $\sigma$ between $f(\mathcal{U})$ and $\hat{f}(\mathcal{U})$ preserves curvature lines $\left(\alpha_{1}^{0} \wedge \alpha_{0}^{1}=\alpha_{2}^{0} \wedge \alpha_{0}^{2}=0\right)$ and is conformal. In the classical terminology [5], this is expressed by saying that $\sigma$ is a conformal Ribaucour congruence, i.e., a Darboux congruence. Accordingly, $\hat{f}$ is called a Darboux transform of $f$.

EXAMPLE 3.2 (Isothermic surfaces of spherical type). In general, the second envelope of the central sphere congruence (the conformal transform) of an isothermic immersion need not be isothermic, see for example [8]. An isothermic immersion is said of spherical type if its central sphere congruence is a Darboux congruence ${ }^{5}$. This is equivalent to

$$
p_{1}+p_{3}=0, \quad p_{2}=0,
$$

and hence, by the structure equations, to

$$
\Delta \psi+e^{2 \psi}=0
$$

As proved in [20], non-minimal isothermic surfaces of spherical type are Darboux transforms of sphere pieces. Moreover, the congruence realizing the Darboux transform is the central sphere congruence. This will complement the discussion in Section 4 to give a complete classification of Darboux transforms of Dupin surfaces.

Let $\mathbf{a}: \mathcal{U} \rightarrow G$ be a principal frame along an isothermic immersion $f: \mathcal{U} \rightarrow \mathbf{S}^{3}$ with connection form $\alpha$, and let $\rho$ be a smooth function such that

$$
\frac{d \rho}{\rho}=-2\left(\alpha_{0}^{0}-l_{1} \alpha_{0}^{1}-l_{2} \alpha_{0}^{2}\right) \text {. }
$$

\footnotetext{
${ }^{4}$ For the related notion of curved flats in symmetric spaces we refer to $[16,8]$.

${ }^{5}$ Equivalently, its central framing is a curved flat framing.
} 
Consider the deformed form

$$
\alpha_{\rho}:=\left(\begin{array}{ccccc}
\alpha_{0}^{0} & \alpha_{1}^{0}+\rho \alpha_{0}^{1} & \alpha_{2}^{0}-\rho \alpha_{0}^{2} & \alpha_{3}^{0} & 0 \\
\alpha_{0}^{1} & 0 & -\alpha_{1}^{2} & -\alpha_{1}^{3} & \alpha_{1}^{0}+\rho \alpha_{0}^{1} \\
\alpha_{0}^{2} & \alpha_{1}^{2} & 0 & -\alpha_{2}^{3} & \alpha_{2}^{0}-\rho \alpha_{0}^{2} \\
0 & \alpha_{1}^{3} & \alpha_{2}^{3} & 0 & \alpha_{3}^{0} \\
0 & \alpha_{0}^{1} & \alpha_{0}^{2} & 0 & -\alpha_{0}^{0}
\end{array}\right)
$$

Since $\alpha_{\rho}$ satisfies the Maurer-Cartan integrability condition

$$
d \alpha_{\rho}=-\alpha_{\rho} \wedge \alpha_{\rho},
$$

$\alpha_{\rho}=\mathbf{a}_{\rho}{ }^{-1} d \mathbf{a}_{\rho}$ for some map $\mathbf{a}_{\rho}: \mathcal{U} \rightarrow G$, unique up to left multiplication by a constant element of $G$. Let $V_{0} \in \mathbf{R}_{1}^{5}$ be any constant vector. Then

$$
V=\mathbf{a}_{\rho}{ }^{-1} V_{0}: \mathcal{U} \rightarrow \mathbf{R}_{1}^{5}
$$

is a solution of

$$
d V=-\alpha_{\rho} V .
$$

Any solution of (21) is obtained in this way. In particular, a solution $V: \mathcal{U} \rightarrow \mathcal{L}^{+}$of $(21)$ is completely determined by a solution $\mathbf{a}_{\rho}: \mathcal{U} \rightarrow G$ of the equation $d \mathbf{a}_{\rho}=\mathbf{a}_{\rho} \alpha_{\rho}$ and a constant vector $V_{0} \in \mathcal{L}^{+}$.

Let $V={ }^{t}\left(v^{0}, \ldots, v^{4}\right): \mathcal{U} \rightarrow \mathcal{L}^{+}$be a solution of $(21)$ for a fixed $\rho$. It is not difficult to see that, generically, the zero locus of $v^{4}$ is discrete ${ }^{6}$. Away from this discrete set, we consider the change of frame given by

$$
g^{+}(V)=\left(\begin{array}{ccc}
\left(v^{4}\right)^{-1} & \left(v^{4}\right)^{-1} t v & v^{0} \\
0 & I_{3} & v \\
0 & 0 & v^{4}
\end{array}\right), \quad v={ }^{t}\left(v^{1}, v^{2}, v^{3}\right)
$$

LEMMA 3.3. The frame $\mathbf{a} g^{+}(V)$ is a curved flat framing and the mapping $\mathbf{a} V$ from $\mathcal{U}$ into the light-cone $\mathcal{L}^{+}$represents a Darboux transform of $f$.

Proof. The proof follows immediately by looking at the corresponding Maurer-Cartan form

$$
\alpha^{+}=\left(g^{+}\right)^{-1} d g^{+}+\left(g^{+}\right)^{-1} \alpha g^{+},
$$

which takes the form

$$
\left(\begin{array}{ccccc}
0 & -v^{4} \rho \alpha_{0}^{1} & v^{4} \rho \alpha_{0}^{2} & 0 & 0 \\
\frac{\alpha_{0}^{1}}{v^{4}} & 0 & -\alpha_{1}^{2}+\frac{v^{2}}{v^{4}} \alpha_{0}^{1}-\frac{v^{1}}{v^{4}} \alpha_{0}^{2} & -\alpha_{1}^{3}+\frac{v^{3}}{v^{4}} \alpha_{0}^{1} & -v^{4} \rho \alpha_{0}^{1} \\
\frac{\alpha_{0}^{2}}{v^{4}} & \alpha_{1}^{2}-\frac{v^{2}}{v^{4}} \alpha_{0}^{1}+\frac{v^{1}}{v^{4}} \alpha_{0}^{2} & 0 & -\alpha_{2}^{3}+\frac{v^{3}}{v^{4}} \alpha_{0}^{2} & v^{4} \rho \alpha_{0}^{2} \\
0 & \alpha_{1}^{3}-\frac{v^{3}}{v^{4}} \alpha_{0}^{1} & \alpha_{2}^{3}-\frac{v^{3}}{v^{4}} \alpha_{0}^{2} & 0 & 0 \\
0 & \frac{\alpha_{0}^{1}}{v^{4}} & \frac{\alpha_{0}^{2}}{v^{4}} & 0 & 0
\end{array}\right) \text {. - . }
$$

We now apply the above construction to the Euclidean and central frames.

\footnotetext{
${ }^{6}$ Clearly, the locus $\left\{v^{4}=0\right\}$ is contained in $\left\{v^{1}=v^{2}=v^{3}=0\right\}$. From (21), it follows that $d v^{1} \wedge d v^{2} \mid\left\{v^{1}=v^{2}=v^{3}=0\right\} \neq 0$. By the inverse function theorem, we then conclude that $\left\{v^{1}=v^{2}=v^{3}=0\right\}$, if not empty, is made of isolated points.
} 
EXAMPLE 3.4 (Darboux transforms via Euclidean frames). Let $f$ be an isothermic immersion and let $\mathbf{e}=\left(e_{0}, \ldots, e_{4}\right)$ denote the Euclidean principal frame along $f$ with connection form $\eta$. In this case the first order system (21) coincides with the Darboux fundamental differential system ${ }^{7}$ ([3], p. 99)

$$
d\left(\begin{array}{c}
v^{0} \\
v^{1} \\
v^{2} \\
v^{3} \\
v^{4}
\end{array}\right)=-\left(\begin{array}{ccccc}
0 & m e^{-\varphi} d x & -m e^{-\varphi} d y & 0 & 0 \\
e^{\varphi} d x & 0 & -\eta_{1}^{2} & -k_{1} e^{\varphi} d x & m e^{-\varphi} d x \\
e^{\varphi} d y & \eta_{1}^{2} & 0 & -k_{2} e^{\varphi} d y & -m e^{-\varphi} d y \\
0 & k_{1} e^{\varphi} d x & k_{2} e^{\varphi} d y & 0 & 0 \\
0 & e^{\varphi} d x & e^{\varphi} d y & 0 & 0
\end{array}\right)\left(\begin{array}{c}
v^{0} \\
v^{1} \\
v^{2} \\
v^{3} \\
v^{4}
\end{array}\right)
$$

for a constant $m$. Then, $\mathbf{e} g^{+}(V)$ is a curved flat framing and the stereographic projection from $\left[\epsilon_{4}\right]$ of its second envelope $\mathbf{e} g^{+}(V)\left(\epsilon_{4}\right)$ gives the classical Darboux transform $D_{m}$ of $f[3]$. By construction $D_{m}(f)$ is represented by the mapping

$$
\mathbf{e} V=v^{0} e_{0}+v^{j} e_{j}+v^{4} \epsilon_{4}
$$

into the light-cone. Since $\mathbf{e}$ is Euclidean, away from the set of isolated points where $v^{0}$ vanishes,

$$
D_{m}(f)=f+\frac{v^{j}}{v^{0}} a_{j}(f), \quad j=1,2,3,
$$

where $\left(a_{1}(f), a_{2}(f), a_{3}(f)\right)$ is the principal framing of $f$ (cf. [3], p. 100, eq. (7)).

EXAMPLE 3.5 (Darboux transforms via central frames). Let $\mathbf{b}$ be the central frame along an isothermic immersion $f: \mathcal{U} \rightarrow \mathbf{S}^{3}$. It is easily checked that $\rho=m e^{-2 \psi}$ for a real constant $m$, and that the deformed connection form $\beta_{\rho}=: \beta(m)$ is given by (17). Let $V: \mathcal{U} \rightarrow \mathcal{L}^{+}$be a solution of $d V=-\beta(m) V$, and consider the curved flat framing (cf. Lemma 1)

$$
\hat{\mathbf{b}}:=\mathbf{b} g^{+}(V): \mathcal{U} \subset \mathbf{R}^{2} \rightarrow G .
$$

The first envelope $\hat{\mathbf{b}}\left(\epsilon_{0}\right)$ represents the original immersion $f$. Let $d_{m}(f):=\left[\hat{\mathbf{b}}\left(\epsilon_{4}\right)\right]$ denote the Darboux transform of $f$. The Calapso potential of $d_{m}(f)$ can be computed as follows. Suppose $m>0$ and consider $\tilde{\mathbf{b}}:=\left(\hat{b}_{4},-\hat{b}_{1}, \hat{b}_{2}, \hat{b}_{3}, \hat{b}_{0}\right)$. $\mathbf{b}$ is a principal frame along $d_{m}(f)$ whose Maurer-Cartan form $\tilde{\beta}$ is of the form

$$
\left(\begin{array}{ccccc}
0 & -\frac{e^{\psi}}{v^{4}} d x & \frac{e^{\psi}}{v^{4}} d y & 0 & 0 \\
v^{4} m e^{-\psi} d x & 0 & \beta_{1}^{2}-\frac{v^{2}}{v^{4}} e^{\psi} d x+\frac{v^{1}}{v^{4}} e^{\psi} d y & \left(1-\frac{v^{3}}{v^{4}}\right) e^{\psi} d x & -\frac{e^{\psi}}{v^{4}} d x \\
v^{4} m e^{-\psi} d y & -\beta_{1}^{2}+\frac{v^{2}}{v^{4}} e^{\psi} d x-\frac{v^{1}}{v^{4}} e^{\psi} d y & 0 & \left(1+\frac{v^{3}}{v^{4}}\right) e^{\psi} d y & \frac{e^{\psi}}{v^{4}} d y \\
0 & -\left(1-\frac{v^{3}}{v^{4}}\right) e^{\psi} d x & -\left(1+\frac{v^{3}}{v^{4}}\right) e^{\psi} d y & 0 & 0 \\
0 & v^{4} m e^{-\psi} d x & v^{4} m e^{-\psi} d y & 0 & 0
\end{array}\right) .
$$

Following the procedure illustrated in Example 2.4, we may adapt the principal frame $\tilde{\mathbf{b}}$ further to a central frame. From this we will learn the expression for the Calapso potential

${ }^{7}$ In Bianchi's notations we have

$$
v^{0}=-m \sigma, \quad v^{1}=\lambda, \quad v^{2}=\mu, \quad v^{3}=-w, \quad v^{4}=-\varphi,
$$

where $\lambda, \mu, w, \varphi, \sigma$ are the five transforming functions (funzioni trasformatrici) from $f$ to $D_{m}(f)$. 
of $d_{m}(f)$. The Calapso potential of the Darboux transform $d_{m}(f)$ is computed to be

$$
\tilde{\Phi}=\frac{v^{3}}{v^{4}} \Phi
$$

Note that the zero locus of $v^{3}$ is the umbilic locus of $d_{m}(f)$.

We are then in a position to state:

LEMma 3.6 (Bäcklund's theorem). With the notations introduced above, let $\Phi$ be the Calapso potential of an isothermic immersion $f$ and let $V: \mathcal{U} \rightarrow \mathcal{L}^{+}$be a solution of the completely integrable linear system:

$$
d V=-\beta_{m}(\Phi) V
$$

Then

$$
\Phi_{m}=\frac{v^{3}}{v^{4}} \Phi
$$

is the Calapso potential of the Darboux transform $d_{m}(f)$. The set $\left\{v^{3}=0\right\}$ is the umbilic locus of $d_{m}(f)$.

4. Darboux transforms of Dupin cyclides. Let $f: \mathcal{U} \rightarrow \mathbf{S}^{3}$ be an umbilic free immersion and let $a, c$ denote its principal curvatures. Let $\mathbf{e}$ be a Euclidean principal frame along $f$ and $\mathbf{a}$ be the central frame obtained from e as indicated in Example 2.4. From (4) and (11), we get

$$
q_{1}=\frac{-c_{2}}{2 R}, \quad q_{2}=\frac{-a_{1}}{2 R} .
$$

Thus $f$ is a Dupin cyclide, that is, equations $a_{x}=c_{y}=0$ hold true, if and only if $q_{1}=q_{2}=0$. Using the structure equations, we also get that $f$ is isothermic $\left(p_{2}=0\right)$, and that the Calapso potential is a constant function. Moreover, equations (8), (9) and (14) imply that $p_{1}$ and $p_{3}$ are constant functions such that $1+p_{1}+p_{3}=0$. Without loss of generality, we may assume $\Phi=1$ and set

$$
p_{1}=k=\text { const. }, \quad p_{3}=-(1+k) .
$$

So, the central frame a along a Dupin cyclide $f$ has Maurer-Cartan form

$$
\alpha=\left(\begin{array}{ccccc}
0 & k d x & -(1+k) d y & 0 & 0 \\
d x & 0 & 0 & -d x & k d x \\
d y & 0 & 0 & d y & -(1+k) d y \\
0 & d x & -d y & 0 & 0 \\
0 & d x & d y & 0 & 0
\end{array}\right) .
$$

4.1. The central frame of Dupin cyclides. In order to compute the central frame $\mathbf{a}=\left(a_{0}, \ldots, a_{4}\right)$ of a cyclide $f$, we need to solve the following linear system

$$
\begin{aligned}
d a_{0} & =a_{1} d x+a_{2} d y \\
d a_{1} & =\left(k a_{0}+a_{3}+a_{4}\right) d x \\
d a_{2} & =\left[-(1+k) a_{0}-a_{3}+a_{4}\right] d y \\
d a_{3} & =-a_{1} d x+a_{2} d y \\
d a_{4} & =k a_{1} d x-(1+k) a_{2} d y .
\end{aligned}
$$


Note that

$$
a_{1}=a_{1}(x), \quad a_{2}=a_{2}(y)
$$

and set

$$
a_{1}=\frac{d b_{1}}{d x}, \quad a_{2}=\frac{d b_{2}}{d y}
$$

From (22), it follows that

$$
\begin{aligned}
& a_{0}=b_{1}+b_{2}+A_{0} \\
& a_{3}=-b_{1}+b_{2}+A_{3} \\
& a_{4}=k b_{1}-(1+k) b_{2}+A_{4},
\end{aligned}
$$

and

$$
\begin{aligned}
& \frac{d^{2} b_{1}}{d x^{2}}=(2 k-1) b_{1}+k A_{0}+A_{3}+A_{4} \\
& \frac{d^{2} b_{2}}{d y^{2}}=-(2 k+3) b_{2}-(1+k) A_{0}-A_{3}+A_{4},
\end{aligned}
$$

where $A_{0}, A_{3}, A_{4}$ are constant vectors. The discussion can be reduced to the study of the following three cases:

$$
I:-\frac{1}{2}<k<\frac{1}{2}, \quad I I: k>\frac{1}{2}, \quad I I I: k=\frac{1}{2} .
$$

CASE I: $-\frac{1}{2}<k<\frac{1}{2}$

$$
\begin{aligned}
& b_{1}=C_{1} \cos \sqrt{1-2 k} x+C_{2} \sin \sqrt{1-2 k} x+\Delta_{1}, \\
& b_{2}=C_{3} \cos \sqrt{2 k+3} y+C_{4} \sin \sqrt{2 k+3} y+\Delta_{2},
\end{aligned}
$$

$C_{i}, \Delta_{\alpha}$ constant vectors. Thus

$$
\begin{aligned}
& a_{1}=\sqrt{1-2 k}\left(-C_{1} \sin \sqrt{1-2 k} x+C_{2} \cos \sqrt{1-2 k} x\right) \\
& a_{2}=\sqrt{2 k+3}\left(-C_{3} \sin \sqrt{2 k+3} y+C_{4} \cos \sqrt{2 k+3} y\right)
\end{aligned}
$$

Since $\left\|a_{1}\right\|^{2}=\left\|a_{2}\right\|^{2}=1$ and $\left\langle a_{1}, a_{2}\right\rangle=0$, it follows that

$$
\left\|C_{1}\right\|^{2}=\left\|C_{2}\right\|^{2}=\frac{1}{1-2 k}, \quad\left\|C_{3}\right\|^{2}=\left\|C_{4}\right\|^{2}=\frac{1}{2 k+3}, \quad\left\langle C_{i}, C_{j}\right\rangle=0, i \neq j .
$$

We may assume that

$$
C_{1}=\frac{\epsilon_{1}}{\sqrt{1-2 k}}, \quad C_{2}=\frac{\epsilon_{0}-\epsilon_{4}}{\sqrt{2(1-2 k)}}, \quad C_{3}=\frac{\epsilon_{2}}{\sqrt{2 k+3}}, \quad C_{4}=\frac{\epsilon_{3}}{\sqrt{2 k+3}} .
$$

From (23), (25), (26), and (24), it follows that

$$
\begin{aligned}
a_{0}= & \frac{2+\sqrt{2 k+3} \sin \sqrt{1-2 k} x}{\sqrt{2(1-2 k)(2 k+3)}} \epsilon_{0}+\frac{\cos \sqrt{1-2 k} x}{\sqrt{1-2 k}} \epsilon_{1}+\frac{\cos \sqrt{2 k+3} y}{\sqrt{2 k+3}} \epsilon_{2} \\
& +\frac{\sin \sqrt{2 k+3} y}{\sqrt{2 k+3}} \epsilon_{3}+\frac{2-\sqrt{2 k+3} \sin \sqrt{1-2 k} x}{\sqrt{2(1-2 k)(2 k+3)}} \epsilon_{4}
\end{aligned}
$$




$$
\begin{aligned}
a_{1}= & \frac{\cos \sqrt{1-2 k} x}{\sqrt{2}} \epsilon_{0}-\sin \sqrt{1-2 k} x \epsilon_{1}-\frac{\cos \sqrt{1-2 k} x}{\sqrt{2}} \epsilon_{4} \\
a_{2}= & -\sin \sqrt{2 k+3} y \epsilon_{2}+\cos \sqrt{2 k+3} y \epsilon_{3}, \\
a_{3}= & \frac{-(2 k+1)-\sqrt{2 k+3} \sin \sqrt{1-2 k} x}{\sqrt{2(1-2 k)(2 k+3)}} \epsilon_{0}-\frac{\cos \sqrt{1-2 k} x}{\sqrt{1-2 k}} \epsilon_{1}+\frac{\cos \sqrt{2 k+3} y}{\sqrt{2 k+3}} \epsilon_{2} \\
& +\frac{\sin \sqrt{2 k+3} y}{\sqrt{2 k+3}} \epsilon_{3}+\frac{-(2 k+1)+\sqrt{2 k+3} \sin \sqrt{1-2 k} x}{\sqrt{2(1-2 k)(2 k+3)}} \epsilon_{4}, \\
a_{4}= & \frac{1+k \sqrt{2 k+3} \sin \sqrt{1-2 k} x}{\sqrt{2(1-2 k)(2 k+3)}} \epsilon_{0}+\frac{k \cos \sqrt{1-2 k} x}{\sqrt{1-2 k}} \epsilon_{1}-\frac{(1+k) \cos \sqrt{2 k+3} y}{\sqrt{2 k+3}} \epsilon_{2} \\
& -\frac{(1+k) \sin \sqrt{2 k+3} y}{\sqrt{2 k+3}} \epsilon_{3}+\frac{1-k \sqrt{2 k+3} \sin \sqrt{1-2 k} x}{\sqrt{2(1-2 k)(2 k+3)}} \epsilon_{4} .
\end{aligned}
$$

This yields

LEMMA 4.1. For $-\frac{1}{2}<k<\frac{1}{2}$, the Dupin cyclide given by the stereographic projection of $f(\mathcal{U})$ is Möbius equivalent to a torus of revolution in $\mathbf{E}^{3}$.

CASE II: $k>\frac{1}{2}$

$$
\begin{aligned}
& b_{1}=\Gamma_{1} \cosh \sqrt{2 k-1} x+\Gamma_{2} \sinh \sqrt{2 k-1} x+\frac{k A_{0}+A_{3}+A_{4}}{1-2 k}, \\
& b_{2}=L_{1} \cos \sqrt{2 k+3} y+L_{2} \sin \sqrt{2 k+3} y+\frac{-(1+k) A_{0}-A_{3}+A_{4}}{2 k+3},
\end{aligned}
$$

$\Gamma_{\alpha}, L_{\alpha}$ constant vectors. Then

$$
\begin{aligned}
& a_{1}=\sqrt{2 k-1}\left(\Gamma_{1} \sinh \sqrt{2 k-1} x+\Gamma_{2} \cosh \sqrt{2 k-1} x\right) \\
& a_{2}=-\sqrt{2 k+3}\left(L_{1} \sin \sqrt{2 k+3} y-L_{2} \cos \sqrt{2 k+3} y\right)
\end{aligned}
$$

Since $\left\|a_{1}\right\|^{2}=\left\|a_{2}\right\|^{2}=1$ and $\left\langle a_{1}, a_{2}\right\rangle=0$, it follows that

$$
\begin{aligned}
& \left\|\Gamma_{1}\right\|^{2}=\frac{-1}{2 k-1}, \quad\left\|\Gamma_{2}\right\|^{2}=\frac{1}{2 k-1}, \quad\left\langle\Gamma_{1}, \Gamma_{2}\right\rangle=0, \\
& \left\|L_{1}\right\|^{2}=\left\|L_{2}\right\|^{2}=\frac{1}{2 k+3}, \quad\left\langle L_{1}, L_{2}\right\rangle=0, \quad\left\langle L_{i}, \Gamma_{j}\right\rangle=0,
\end{aligned}
$$

and we may assume

$$
\Gamma_{1}=\frac{\epsilon_{0}+\epsilon_{4}}{\sqrt{2(2 k-1)}}, \quad \Gamma_{2}=\frac{\epsilon_{1}}{\sqrt{2 k-1}}, \quad L_{1}=\frac{\epsilon_{2}}{\sqrt{2 k+3}}, \quad L_{2}=\frac{\epsilon_{3}}{\sqrt{2 k+3}} .
$$

By imposing the other conditions

$$
\left\|a_{0}\right\|^{2}=0, \quad\left\|a_{3}\right\|^{2}=1, \quad\left\|a_{4}\right\|^{2}=0 \quad\left\langle a_{i}, a_{j}\right\rangle=0, \quad i \neq j, i, j=0,3,4,
$$

we obtain the following expressions for $a_{0}, a_{1}, a_{2}, a_{3}, a_{4}$ :

$$
\begin{aligned}
a_{0}= & \frac{\sqrt{2 k+3} \cosh \sqrt{2 k-1} x+2}{\sqrt{2(2 k-1)(2 k+3)}} \epsilon_{0}+\frac{\sinh \sqrt{2 k-1} x}{\sqrt{2 k-1}} \epsilon_{1}+\frac{\cos \sqrt{2 k+3} y}{\sqrt{2 k+3}} \epsilon_{2} \\
& +\frac{\sin \sqrt{2 k+3} y}{\sqrt{2 k+3}} \epsilon_{3}+\frac{\sqrt{2 k+3} \cosh \sqrt{2 k-1} x-2}{\sqrt{2(2 k-1)(2 k+3)}} \epsilon_{4},
\end{aligned}
$$




$$
\begin{aligned}
a_{1}= & \frac{\sinh \sqrt{2 k-1} x}{\sqrt{2}} \epsilon_{0}+\cosh \sqrt{2 k-1} x \epsilon_{1}+\frac{\sinh \sqrt{2 k-1} x}{\sqrt{2}} \epsilon_{4} \\
a_{2}= & -\sin \sqrt{2 k+3} y \epsilon_{2}+\cos \sqrt{2 k+3} y \epsilon_{3} \\
a_{3}= & -\frac{\sqrt{2 k+3} \cosh \sqrt{2 k-1} x+(2 k+1)}{\sqrt{2(2 k-1)(2 k+3)}} \epsilon_{0}-\frac{\sinh \sqrt{2 k-1} x}{\sqrt{2 k-1}} \epsilon_{1}+\frac{\cos \sqrt{2 k+3} y}{\sqrt{2 k+3}} \epsilon_{2} \\
& +\frac{\sin \sqrt{2 k+3} y}{\sqrt{2 k+3}} \epsilon_{3}-\frac{\sqrt{2 k+3} \cosh \sqrt{2 k-1} x-(2 k+1)}{\sqrt{2(2 k-1)(2 k+3)}} \epsilon_{4}, \\
a_{4}= & \frac{k \sqrt{2 k+3} \cosh \sqrt{2 k-1} x+1}{\sqrt{2(2 k-1)(2 k+3)}} \epsilon_{0}+\frac{k \sinh \sqrt{2 k-1} x}{\sqrt{2 k-1}} \epsilon_{1}-\frac{(k+1) \cos \sqrt{2 k+3} y}{\sqrt{2 k+3}} \epsilon_{2} \\
& -\frac{(k+1) \sin \sqrt{2 k+3} y}{\sqrt{2 k+3}} \epsilon_{3}+\frac{k \sqrt{2 k+3} \cosh \sqrt{2 k-1} x-1}{\sqrt{2(2 k-1)(2 k+3)}} \epsilon_{4} .
\end{aligned}
$$

From this we have

Lemma 4.2. For $k>\frac{1}{2}$, the Dupin cyclide given by the stereographic projection of $f(\mathcal{U})$ is a Möbius equivalent to a circular cone in $\mathbf{E}^{3}$.

CASE III: $\quad k=\frac{1}{2}$. In this case

$$
\begin{aligned}
& b_{1}=\frac{1}{2}\left(\frac{1}{2} A_{0}+A_{3}+A_{4}\right) x^{2}+\Gamma_{1} x+\Gamma_{2} \\
& b_{2}=L_{1} \cos 2 y+L_{2} \sin 2 y+\frac{1}{4}\left(-\frac{3}{2} A_{0}-A_{3}+A_{4}\right) .
\end{aligned}
$$

According to (23) and the orthogonality conditions we may choose the constant vectors of integration so that

$$
\begin{aligned}
& a_{0}=\frac{1}{2}\left(x^{2}+\frac{1}{4}\right) \epsilon_{0}+x \epsilon_{1}+\frac{1}{2}\left(\cos 2 y \epsilon_{2}+\sin 2 y \epsilon_{3}\right)+\epsilon_{4}, \\
& a_{1}=x \epsilon_{0}+\epsilon_{1}, \\
& a_{2}=-\sin 2 y \epsilon_{2}+\cos 2 y \epsilon_{3}, \\
& a_{3}=-\frac{1}{2}\left(x^{2}-\frac{3}{4}\right) \epsilon_{0}-x \epsilon_{1}+\frac{1}{2}\left(\cos 2 y \epsilon_{2}+\sin 2 y \epsilon_{3}\right)-\epsilon_{4}, \\
& a_{4}=\frac{1}{4}\left(x^{2}+\frac{9}{4}\right) \epsilon_{0}+\frac{1}{2} x \epsilon_{1}-\frac{3}{4}\left(\cos 2 y \epsilon_{2}+\sin 2 y \epsilon_{3}\right)+\frac{1}{2} \epsilon_{4}
\end{aligned}
$$

In this case we have

Lemma 4.3. For $k=\frac{1}{2}$, the Dupin cyclide given by the stereographic projection of $f(\mathcal{U})$ is Möbius equivalent to a circular cylinder in $\mathbf{E}^{3}$.

REMARK 4.4. As already observed, from the previous formulae we can conclude that Dupin cyclides arise as T-transforms of the circular cylinder. 
4.2. The Darboux transformation of Dupin cyclides. The linear system (21) becomes

$$
\begin{aligned}
d v^{0} & =-\tilde{k} v^{1} d x+(1+\tilde{k}) v^{2} d y \\
d v^{1} & =-v^{0} d x+v^{3} d x-\tilde{k} v^{4} d x \\
d v^{2} & =-v^{0} d y-v^{3} d y+(1+\tilde{k}) v^{4} d y \\
d v^{3} & =-v^{1} d x+v^{2} d y \\
d v^{4} & =-v^{1} d x-v^{2} d y,
\end{aligned}
$$

where $\tilde{k}=k+m$. Note that

$$
\begin{aligned}
& v^{1}=v^{1}(x) \\
& v^{2}=v^{2}(y) .
\end{aligned}
$$

Let $u^{1}, u^{2}$ be functions such that $d u^{1}=v^{1} d x, d u^{2}=v^{2} d y$. Then

$$
\begin{aligned}
& d v^{0}=-\tilde{k} d u^{1}+(1+\tilde{k}) d u^{2} \\
& d v^{3}=-d u^{1}+d u^{2} \\
& d v^{4}=-d u^{1}-d u^{2},
\end{aligned}
$$

that is

$$
\begin{aligned}
& v^{0}=-\tilde{k} u^{1}+(1+\tilde{k}) u^{2}+\alpha^{0} \\
& v^{3}=-u^{1}+u^{2}+\alpha^{3} \\
& v^{4}=-u^{1}-u^{2}+\alpha^{4}
\end{aligned}
$$

$\alpha^{0}, \alpha^{3}, \alpha^{4}$ constants. Combining (27) and (28) yields ODEs for $u^{1}$ and $u^{2}$ :

$$
\begin{aligned}
& \frac{d^{2} u^{1}}{d x^{2}}+(1-2 \tilde{k}) u^{1}=-\alpha^{0}+\alpha^{3}-\tilde{k} \alpha^{4} \\
& \frac{d^{2} u^{2}}{d y^{2}}+(3+2 \tilde{k}) u^{2}=-\alpha^{0}-\alpha^{3}+(1+\tilde{k}) \alpha^{4} .
\end{aligned}
$$

Five cases may occur:

$I: \tilde{k}>\frac{1}{2}, \quad I I: \tilde{k}=\frac{1}{2}, \quad I I I:-\frac{3}{2}<\tilde{k}<\frac{1}{2}, \quad I V: \tilde{k}=-\frac{3}{2}, \quad V: \tilde{k}<-\frac{3}{2}$.

Case I.

$$
\begin{aligned}
& u^{1}=c_{1} \cosh \sqrt{2 \tilde{k}-1} x+c_{2} \sinh \sqrt{2 \tilde{k}-1} x+\frac{\left(-\alpha^{0}+\alpha^{3}-\tilde{k} \alpha^{4}\right)}{1-2 \tilde{k}} \\
& u^{2}=l_{1} \cos \sqrt{2 \tilde{k}+3} y+l_{2} \sin \sqrt{2 \tilde{k}+3} y+\frac{\left(-\alpha^{0}-\alpha^{3}+(1+\tilde{k}) \alpha^{4}\right)}{3+2 \tilde{k}}
\end{aligned}
$$

Case II.

$$
\begin{aligned}
& u^{1}=\frac{1}{2}\left(-\alpha^{0}+\alpha^{3}-\frac{\alpha^{4}}{2}\right) x^{2}+c_{1} x+c_{2} \\
& u^{2}=l_{1} \cos 2 y+l_{2} \sin 2 y+\frac{1}{4}\left(-\alpha^{0}-\alpha^{3}+\frac{3}{2} \alpha^{4}\right)
\end{aligned}
$$

CASE III.

$$
\begin{aligned}
& u^{1}=c_{1} \cos \sqrt{1-2 \tilde{k}} x+c_{2} \sin \sqrt{1-2 \tilde{k}} x+\frac{\left(-\alpha^{0}+\alpha^{3}-\tilde{k} \alpha^{4}\right)}{1-2 \tilde{k}} \\
& u^{2}=l_{1} \cos \sqrt{2 \tilde{k}+3} y+l_{2} \sin \sqrt{2 \tilde{k}+3} y+\frac{\left(-\alpha^{0}-\alpha^{3}+(1+\tilde{k}) \alpha^{4}\right)}{3+2 \tilde{k}}
\end{aligned}
$$


CASE IV.

$$
\begin{aligned}
& u^{1}=c_{1} \cos 2 x+c_{2} \sin 2 x+\frac{1}{4}\left(-\alpha^{0}+\alpha^{3}+\frac{3}{2} \alpha^{4}\right) \\
& u^{2}=\frac{1}{2}\left(-\alpha^{0}-\alpha^{3}-\frac{\alpha^{4}}{2}\right) y^{2}+l_{1} x+l_{2}
\end{aligned}
$$

CASE V.

$$
\begin{aligned}
& u^{1}=c_{1} \cos \sqrt{1-2 \tilde{k}} x+c_{2} \sin \sqrt{1-2 \tilde{k}} x+\frac{\left(-\alpha^{0}+\alpha^{3}-\tilde{k} \alpha^{4}\right)}{1-2 \tilde{k}} \\
& u^{2}=l_{1} \cosh \sqrt{-2 \tilde{k}-3} y+l_{2} \sinh \sqrt{-2 \tilde{k}-3} y+\frac{\left(-\alpha^{0}-\alpha^{3}+(1+\tilde{k}) \alpha^{4}\right)}{3+2 \tilde{k}}
\end{aligned}
$$

REMARK 4.5. Starting from the solution $\Phi \equiv 1$ of Calapso's equation, according to Bäcklund's theorem (Lemma 3.6), we obtain new solutions

$$
\Phi=\frac{-u^{1}+u^{2}+\alpha^{3}}{u^{1}+u^{2}-\alpha^{4}}
$$

of the Calapso equation.

If $V={ }^{t}\left(v^{0}, \ldots, v^{4}\right)$ is a solution of (27), then the Darboux transform of $f$ associated with $V$ is represented by the mapping $\mathbf{a} V: \mathcal{U}-\left\{v^{4}=0\right\} \rightarrow \mathcal{L}^{+}$given by $\left(-\tilde{k} u^{1}+(1+\tilde{k}) u^{2}+\alpha^{0}\right) a_{0}+\frac{d u^{1}}{d x} a_{1}+\frac{d u^{2}}{d y} a_{2}+\left(-u^{1}+u^{2}+\alpha^{3}\right) a_{3}+\left(-u^{1}-u^{2}+\alpha^{4}\right) a_{4}$. The constants of integration must be chosen so that $\|\mathbf{a} V\|^{2}=0$.

REMARK 4.6. Bernstein [2] constructs explicit examples of isothermic tori which are neither conformally equivalent to tori of revolution nor to constant mean curvature tori in space forms. These tori are obtained by carefully analyzing the Darboux transforms of the torus of revolution computed above. They have spherical curvature lines and, in contrast to the original surface, can have umbilics; either isolated umbilics or lines of umbilics can occur. The proof uses the expression for the Calapso potential of the Darboux transforms, computed according to Bäcklund's theorem (Lemma 3.6), in combination with equation (18) whose solutions correspond to constant mean curvature surfaces in 3-dimensional space forms (cf. Example 2.8).

The existence of constant mean curvature Darboux transforms of a constant mean curvature surface is a classical result of Bianchi [3], [19]. In particular, the constant mean curvature Darboux transforms of the cylinder have been considered in [19]. They are related to Sterling-Wente's constant mean curvature multibubbletons which in turn are obtained from the cylinder via the Bianchi-Bäcklund transformation [25]. In fact, Hertrich-Jeromin-Pedit [19] prove that any Bianchi-Bäcklund transform of a constant mean curvature surface is a Darboux transform. For recent studies on constant mean curvature Darboux transforms of constant mean curvature surfaces in space forms and their relations with the classical special isothermic surfaces of Darboux [3] we refer the reader to $[22]$. 
5. Superposition and permutability. As another application of our construction we provide a superposition formula for iterated Darboux transforms of a given isothermic surface and give a proof of Bianchi's permutability theorem [3]. This formula is particularly useful for the explicit calculation of multisoliton surfaces (see figures and [22]).

Let $\mathbf{a}^{(1)}, \mathbf{a}^{(2)}: \mathcal{U} \rightarrow G$ be two curved flat frames having the same isothermic immersion $f=\left[a_{0}^{(1)}\right]=\left[a_{0}^{(2)}\right]: \mathcal{U} \rightarrow \mathbf{S}^{3}$ as first envelope and with second envelopes $f^{(1)}$ and $f^{(2)}$, respectively. Let $\alpha^{(1)}=\left(\alpha_{j}^{i}\right), \alpha^{(2)}=\left(\tilde{\alpha}_{j}^{i}\right)$ be the corresponding connection forms ${ }^{8}$.
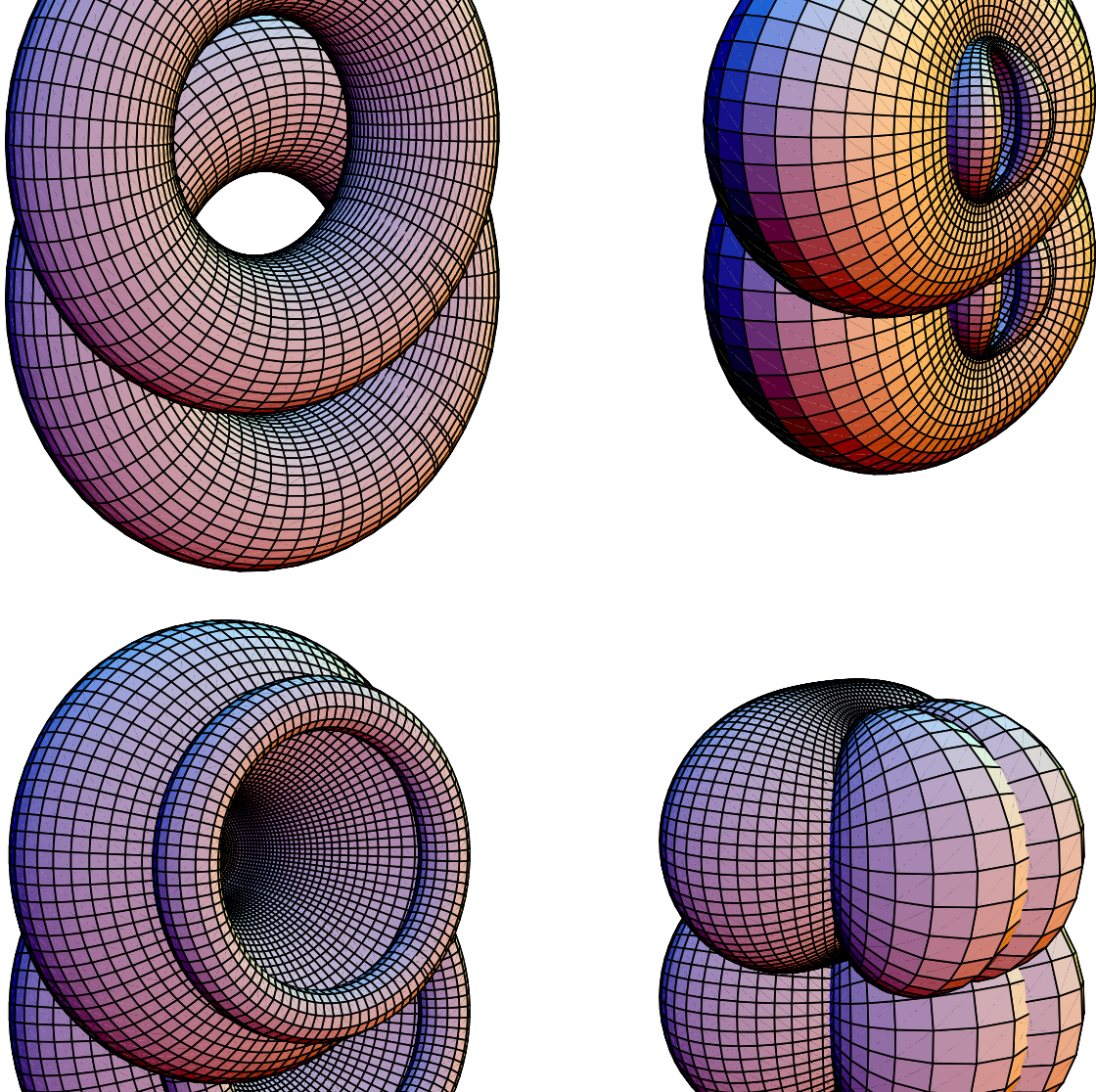

Fig. 1. Darboux transforms of the torus

${ }^{8}\left(\alpha_{j}^{i}\right)$ can be described by

$$
\begin{aligned}
& \alpha_{0}^{0}=\alpha_{0}^{3}=\alpha_{3}^{0}=0 \\
& \alpha_{0}^{1}=e^{u} d x, \quad \alpha_{0}^{2}=e^{u} d y, \quad \alpha_{1}^{0}=m e^{-u} d x, \quad \alpha_{2}^{0}=-m e^{-u} d y \\
& \alpha_{1}^{3}=H_{1} e^{u} d x, \quad \alpha_{2}^{3}=H_{2} e^{u} d y,
\end{aligned}
$$

for functions $H_{1}, H_{2}, u: \mathcal{U} \rightarrow \mathbf{R}$, and some constant $m$ (see [8]). 

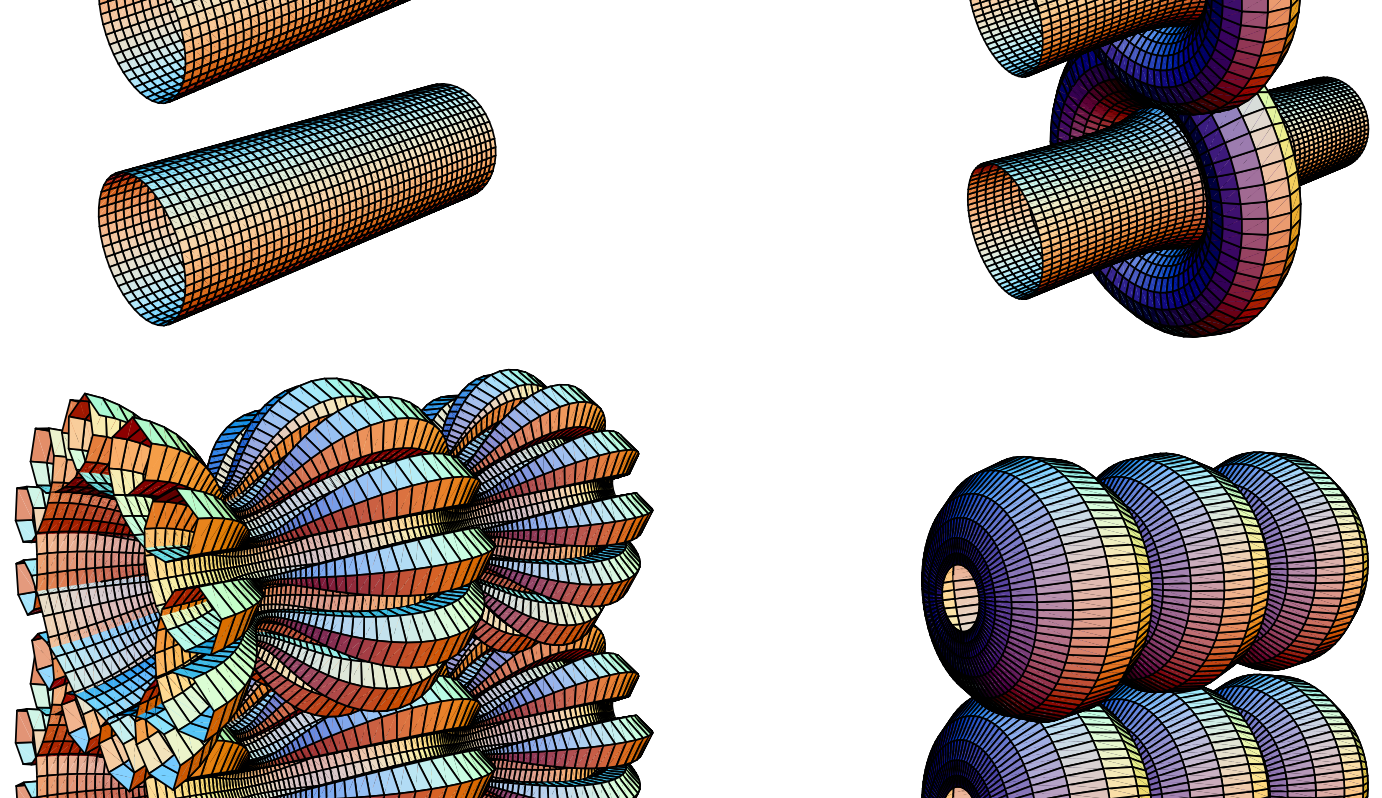

Fig. 2. Darboux transforms of the cylinder

The deformed forms associated with $\alpha^{(1)}$ are given by

$$
\alpha_{\lambda}^{(1)}:=\left(\begin{array}{ccccc}
0 & \lambda \alpha_{1}^{0} & \lambda \alpha_{2}^{0} & 0 & 0 \\
\alpha_{0}^{1} & 0 & -\alpha_{1}^{2} & -\alpha_{1}^{3} & \lambda \alpha_{1}^{0} \\
\alpha_{0}^{2} & \alpha_{1}^{2} & 0 & -\alpha_{2}^{3} & \lambda \alpha_{2}^{0} \\
0 & \alpha_{1}^{3} & \alpha_{2}^{3} & 0 & 0 \\
0 & \alpha_{0}^{1} & \alpha_{0}^{2} & 0 & 0
\end{array}\right)
$$

for some constant $\lambda$. Similarly for $\alpha^{(2)}$.

According to Section 3, there is a solution $V={ }^{t}\left(v^{0}, \ldots, v^{4}\right): \mathcal{U} \rightarrow \mathcal{L}^{+}$of the integrable linear system

$$
d V=-\alpha_{\lambda}^{(1)} V
$$

for some $\lambda$, such that, on the complement of the discrete zero locus of $v^{4}$, the frames $\mathbf{a}^{(1)}, \mathbf{a}^{(2)}$ are related by the gauge change

$$
\mathbf{a}^{(2)}=\mathbf{a}^{(1)} g^{+}(V)
$$

Next, away from the set of isolated points where $v^{0}$ vanishes, define the mapping $g_{\lambda}^{-}(V)$ : $\mathcal{U} \rightarrow G$ by

$$
g_{\lambda}^{-}(V)=\left(\begin{array}{ccc}
(\lambda)^{-1} v^{0} & 0 & 0 \\
v & I_{3} & 0 \\
\lambda v^{4} & \left(v^{0}\right)^{-1} \lambda^{t} v & \lambda\left(v^{0}\right)^{-1}
\end{array}\right)
$$

We have 
LEMMA 5.1. $\overline{\mathbf{a}}^{(1)}:=\mathbf{a}^{(1)} g_{\lambda}^{-}(V)$ is a curved flat framing whose second envelope $\left[\bar{a}_{4}^{(1)}\right]=$ $f^{(1)}$. Its connection form is given by

$$
\left(\begin{array}{ccccc}
0 & \frac{\lambda}{v^{0}} \alpha_{1}^{0} & \frac{\lambda}{v^{0}} \alpha_{2}^{0} & 0 & 0 \\
v^{0}\left(\frac{1}{\lambda}-1\right) \alpha_{0}^{1} & 0 & -\alpha_{1}^{2}+\lambda \frac{v^{2}}{v^{0}} \alpha_{1}^{0}-\lambda \frac{v^{1}}{v^{0}} \alpha_{2}^{0} & -\alpha_{1}^{3}+\lambda \frac{v^{3}}{v^{0}} \alpha_{1}^{0} & \frac{\lambda}{v^{0}} \alpha_{1}^{0} \\
v^{0}\left(\frac{1}{\lambda}-1\right) \alpha_{0}^{2} & \alpha_{1}^{2}-\lambda \frac{v^{2}}{v^{0}} \alpha_{1}^{0}+\lambda \frac{v^{1}}{v^{0}} \alpha_{2}^{0} & 0 & -\alpha_{2}^{3}+\lambda \frac{v^{3}}{v^{0}} \alpha_{2}^{0} & \frac{\lambda}{v^{0}} \alpha_{2}^{0} \\
0 & \alpha_{1}^{3}-\lambda \frac{v^{3}}{v^{0}} \alpha_{1}^{0} & \alpha_{2}^{3}-\lambda \frac{v^{3}}{v^{0}} \alpha_{2}^{0} & 0 & 0 \\
0 & v^{0}\left(\frac{1}{\lambda}-1\right) \alpha_{0}^{1} & v^{0}\left(\frac{1}{\lambda}-1\right) \alpha_{0}^{2} & 0 & 0
\end{array}\right) .
$$

Definition 5.2. Let $f^{\prime}=\left[\bar{a}_{0}^{(1)}\right]$ be the first envelope of $\overline{\mathbf{a}}^{(1)}$. We say that the isothermic map $f^{\prime}$ is the superposition of the two Darboux transforms $f^{(1)}$ and $f^{(2)}$ of $f$. We write

$$
f^{\prime}=f^{(1)} *_{f} f^{(2)} .
$$

Proposition 5.3 (Permutability Theorem). If an isothermic immersion $f$ has two Darboux transforms $f^{(1)}$ and $f^{(2)}$, then there is another isothermic immersion $f^{\prime}$ which is a Darboux transform of $f^{(1)}$ and $f^{(2)}$ and is such that

$$
f^{\prime}=f^{(1)} *_{f} f^{(2)}=f^{(2)} *_{f} f^{(1)} .
$$

Proof. We can write

$$
\mathbf{a}^{(1)}=\mathbf{a}^{(2)} g^{+}(V)^{-1}=\mathbf{a}^{(2)} g^{+}(\hat{V}),
$$

where

$$
\hat{V}={ }^{t}\left(v^{0},-\frac{{ }^{t} v}{v^{4}}, \frac{1}{v^{4}}\right) .
$$

By a direct calculation it is easily verified that, if $V: \mathcal{U} \rightarrow \mathcal{L}^{+}$is a solution of (29), then $\hat{V}$ is a solution of

$$
d \hat{V}=-\alpha_{\mu}^{(2)} \hat{V}
$$

where $\mu$ is given by

$$
\mu=\lambda(\mu-1) .
$$

Thus, $f^{(2)} *_{f} f^{(1)}$ is the isothermic map represented by the first column of the framing $\overline{\mathbf{a}}^{(2)}=\mathbf{a}^{(2)} g_{\mu}^{-}(\hat{V})$, where $g_{\mu}^{-(\hat{V})}$ is defined in analogy with (5). It is now easily seen that, if $\hat{V}$ and $\mu$ are related to $V$ and $\lambda$ as in (30) and (31), then $\left[\bar{a}_{0}^{(2)}\right]=\left[\bar{a}_{0}^{(1)}\right]$.

\section{References}

[1] M. Babich and A. Bobenko, Willmore tori with umbilic lines and minimal surfaces in hyperbolic space, Duke Math. J. 72 (1993), 151-185.

[2] H. E. Bernstein, Non-special, non-canal isothermic tori with spherical lines of curvature, Trans. Amer. Math. Soc. 353 (2001), 2245-2274; Isothermic tori with spherical lines of curvature, thesis, Washington University, St. Louis, 1999.

[3] L. Bianchi, Ricerche sulle superficie isoterme e sulla deformazione delle quadriche, Ann. Mat. Pura Appl. (3) 11 (1905), 93-157.

[4] L. Bianchi, Complementi alle ricerche sulle superficie isoterme, Ann. Mat. Pura Appl. (3) 12 (1905), 20-54. 
[5] W. Blaschke, Vorlesungen über Differentialgeometrie und geometrische Grundlagen von Einsteins Relativitätstheorie, B. 3, bearbeitet von G. Thomsen, J. Springer, Berlin, 1929.

[6] M. Brück, X. Du, J. Park and C.-L. Terng, The submanifold geometries associated to Grassmannian systems, e-print math.DG/0006216

[7] F. Burstall, Isothermic surfaces: conformal geometry, Clifford algebras and integrable systems, e-print math.DG/0003096

[8] F. Burstall, U. Hertrich-Jeromin, F. Pedit and U. Pinkall, Curved flats and isothermic surfaces Math. Z. 225 (1997), 199-209.

[9] R. L. Bryant, A duality theorem for Willmore surfaces, J. Differential Geom. 20 (1984), $23-53$.

[10] P. Calapso, Sulla superficie a linee di curvatura isoterme, Rend. Circ. Mat. Palermo 17 (1903), 273-286.

[11] D. Carfi and E. Musso, T-transformations of Willmore isothermic surfaces, Rend. Sem. Mat. Messina Ser. II, suppl. 2000, 257-266.

[12] É. Cartan, Les espaces à connexion conforme, Ann. Soc. Pol. Math. 2 (1923), 171-221.

[13] J. Cieśliński, P. Goldstein and A. Sym, Isothermic surfaces in $\mathbf{E}^{3}$ as soliton surfaces, Physics Letters A 205 (1995), 37-43.

[14] J. Cieśliński, The Darboux-Bianchi transformation for isothermic surfaces. Classical versus the soliton approach, Differential Geom. Appl. 2 (1997), 1-28.

[15] G. Darboux, Sur les surfaces isothermiques, C. R. Acad. Sci. Paris 128 (1899), 1299-1305, Ann. Sci. École Norm. Sup. 3 (1899), 491-508.

[16] D. Ferus and F. Pedit, Curved flats in symmetric spaces, Manuscripta Math. 91 (1996), 445-454.

[17] G. Fubini, Applicabilità proiettiva di due superficie, Rend. Circ. Mat. Palermo 41 (1916), $135-161$.

[18] U. Hertrich-Jeromin, Supplement on curved flats in the space of point pairs and isothermic surfaces: a quaternionic calculus, Documenta Math. J. DMV 2 (1997), 335-350.

[19] U. Hertrich-Jeromin and F. Pedit, Remarks on the Darboux transform of isothermic surfaces, Documenta Math. J. DMV 2 (1997), 313-333.

[20] U. Hertrich-Jeromin, E. Musso and L. Nicolodi, Möbius geometry of surfaces of constant mean curvature 1 in hyperbolic space, Ann. Global Anal. Geom. 19 (2001), 185-205.

[21] E. Musso, Deformazione di superfici nello spazio di Möbius, Rend. Istit. Mat. Univ. Trieste 27 (1995), 25-45.

[22] E. Musso and L. Nicolodi, Special isothermic surfaces and solitons, Contemp. Math. 288 (2001), 129-148.

[23] U. Pinkall and I. Sterling, On the classification of constant mean curvature tori, Ann. of Math. 130 (1989), 407-451.

[24] R. Rothe, Untersuchungen über die Theorie der isothermen Flächen, Berlin thesis, Mayer und Müller, Berlin, 1897.

[25] I. Sterling and H. C. Wente, Existence and classification of constant mean curvature multibubbletons of finite and infinite type, Indiana Univ. Math. J. 42 (1993), 1239-1266.

[26] C. L. Terng and K. Uhlenbeck, Bäcklund's transformations and loop group actions, e-print math.DG/9805074

[27] M. E. Vessiot, Contribution à la géométrie conforme. Théorie des surfaces, Bull. Soc. Math. France 54 (1926), 139-179, 55 (1927), 39-79. 